\title{
Taxes, budgetary rule and majority voting
}

Helmuth Cremer

IDEI and GREMAQ; University of Toulouse

Philippe De Donder

IDEI and GREMAQ; University of Toulouse

Firouz Gahvari

Department of Economics;

University of Illinois at Urbana-Champaign

March 2002

This version: December 2002 


\begin{abstract}
This paper studies majority voting outcomes for a specific class of twodimensional policies. The illustrative policy we consider consists of a tax on an externality-generating good and a budgetary rule specifying the proportions of the tax proceeds that go to wage earners and to capital owners. We show: First, a sequential vote wherein the tax rate is determined first and the budgetary rule second, always possesses an equilibrium and that this equilibrium is the median-endowed individual's most-preferred policy. Second, the reverse sequential choice implies that the median-endowed individual may, but need not, be decisive. Third, the "Shepsle procedure" and the "citizen-candidate" framework also imply that the equilibrium is the policy most favored by the median individual. Fourth, this equilibrium also constitutes, under certain circumstances, the Condorcet winner for the unrestricted simultaneous voting game.
\end{abstract}

Key words: Two dimensional majority voting, political support, existence of a Condorcet winner

JEL Classification: H23, D72. 


\section{Introduction}

In democracies, citizens exercise their influence on public decision making by casting their vote on election day. One important component of such public decisions is the efficiency of the proposal under consideration: the quantity of a public good to be provided, the way its provision is to be organized; or more generally, the effectiveness of a proposed policy to alleviate a market failure. Beyond efficiency, the other important component of any public policy is its redistributive impact. A voters' utility depends not just on the efficiency of a public decision, but also on the impact of the policy on the voter's after-tax income. This suggests that the way the policy is financed plays a fundamental role in its political feasibility.

The (non-)separability between equity and efficiency aspects of policy design has been widely discussed in the public economics literature. It is by now well known that while efficiency and equity considerations can be dealt with separately in a (suitably defined) first-best setting, they tend to be interdependent in a second best world. In this paper, we point to yet another source of non-separability, namely the political process and, in particular, multi-dimensional voting procedures. We consider a setting where, absent the political process, the two issues are clearly separated. The policy to be chosen is composed of two elements, one influencing efficiency and the other one redistribution. The model is designed so that the (Pareto) efficient level of the first instrument does not depend on redistribution. However, when a two dimensional vote is considered the policies will interact in a non-trivial way. Moreover the exact nature of interaction will depend on the specific voting procedure considered.

While the point we are making is fairly general, we will cast our formal analysis within a specific bidimensional setting. This is done for concreteness, but also because we belive that the considered setting is of interest in its own right. The public policy is one of correcting a market failure due 
to an externality-generating good, through a tax/subsidy scheme. Many situations may be covered by such a framework, including cases where externalities are positive (e.g. education). The example we concentrate on is that of pollution.

There are several reasons for this. First, environmental policy has received much attention during the past two decades or so, making it an interesting problem to explore from a political economy perspective. A second reason lies in the availability of data on this topic, and particularly on the measurement of externalities created by the consumption of such polluting goods as energy. We plan to test empirically the predictions of our model in future work. Third, we would like to see how our results compare with those of Cremer et al.(2002). There, we have considered an (almost) identical setup but have adopted a different perspective that combines normative and positive considerations. ${ }^{1}$ Finally, our aim is to shed some fresh light on the so-called double dividend hypothesis by casting it in a political economy context. The double dividend hypothesis suggests that the recycling of tax revenues (in particular through reduction in taxes on labor income) is a crucial ingredient of environmental tax policy. We argue that the significance of this aspect is not necessarily linked to the reduction of distortions associated with the pre-existing taxes. Instead, it plays an important role in ensuring political support for a policy which otherwise would be infeasible.

The main features of our model are as follows. We consider a policy that consists of two components: a tax and a budgetary rule. The tax is levied on the consumption of externality generating (polluting) goods. The budgetary rule specifies the way the tax proceeds are redistributed to the polity; in particular, what proportion is directed to the wage earners and what to capital owners. Citizens have their income originating from both sources but at varying shares. We study voting procedures that differ in their

\footnotetext{
${ }^{1}$ Taxes are chosen by the political process as in the current paper, but the budgetary rule is set by some welfare maximizing authority (possibly at an earlier, "constitutional" stage).
} 
timing (simultaneously or sequentially) of the votes on the two components of the environmental policy. The procedures differ also in the restrictions they impose on the set of admissible policies. Preferences are such that the median-endowed individual is decisive when the voting is on one dimension (with the other dimension being fixed). However, when one takes account of the bidimensionality of the decision to be taken, strategic aspects may (but need not) surface $^{2}$.

The main results of the paper are as follows. First, a sequential vote wherein the tax rate is determined first and the budgetary rule second, always possesses an equilibrium and this equilibrium is the median-endowed individual's most-preferred policy. This is due to the fact that one's preferences over the budgetary rule is independent of the value of the tax rate. The preferences are determined solely by the relative shares of the individual's income due to labor and capital sources.

Second, we argue that the reverse sequential choice, in which the budgetary rule is determined first and the tax rate second, entails a more complex interaction between the two decisions. The reason for this is that the budgetary rule affects preferences over the tax rates. We show that in this case the median-endowed individual may, but need not, be decisive. Additionally, we give the necessary conditions on the income distribution for the median person not to be decisive.

Third, we examine the "Shepsle procedure" for simultaneous voting. In this scheme, the decisions on the two dimensions are taken separately but the outcome of each dimension must be consistent with the other to consti-

\footnotetext{
${ }^{2}$ Cremer et al. (2002) have used a similar framework to study the welfare properties of politically viable environmental taxes. They have examined the relationship of the (politically constrained) second-best taxes with their (unconstrained) first-best Pigouvian counterparts. The current paper takes an entirely positive approach and focuses on the various ways that a majority voting outcome can be attained. Another important difference is that Cremer et al. (2002) assume that the budgetary rule is always determined in the first stage and that through two distinct mechanisms: a welfare-maximizing constitutional planner or a majority voting scheme. The tax rate is determined in the second stage via a majority-voting procedure. They also impose certain restrictions on income distribution which we relax here.
} 
tute an equilibrium. We show that in this case too, the equilibrium is the policy most favored by the median-endowed individual. Fourth, we study the simultaneous determination of the tax rate and the budgetary rule while assuming that the vote is taken over the voters' most-preferred policies. ${ }^{3} \mathrm{We}$ show that under this restriction an equilibrium always exists. It is once again given by the median-endowed individual's most-preferred policy.

Fifth, we relax the preceding constraints and allow a simultaneous vote that takes place over all feasible options. The literature on multidimensional voting, starting with Plott (1967), is very gloomy when it comes to the possibility of having an equilibrium (a policy preferred by a majority of voters to any other admissible policy, i.e. a Condorcet winner) in such circumstances. However, these negative results typically rely on the property that feasible options constitute an open set. In our case, we do not have an open set and the existence of a Condorcet winner cannot be ruled out a priori. Indeed, we can infer from our results that if a Condorcet winner exists, it will be the policy that the median-endowed individual prefers most. We describe the circumstances under which this policy can be defeated by a majority of voters, and give examples where a Condorcet winner does or does not exist.

\section{The model ${ }^{4}$}

Individuals are identified by a parameter $\theta$ which is continuously distributed over $[0,1]$ according to the density function, $f(\theta)$. The associated cumulative distribution function is $F(\theta)$. Population size is normalized at one. An individual of type $\theta$ has a total income of $m(\theta)$, with

$$
m(\theta)=w(\theta)+r(\theta)
$$

\footnotetext{
${ }^{3}$ This may be justified by considering that citizen-candidates can not commit to a policy.

${ }^{4}$ Some of the material reported in this Section, as well as in Sections 3-4, are contained also in Cremer et al. (2001). This is done to make the paper self-contained and to avoid cumbersome cross referencing.
} 
where $w(\theta)$ is labor and $r(\theta)$ is capital income. All sources of income are exogenous and individuals are ranked such that $m^{\prime}(\theta)>0$, i.e., total income increases with $\theta$. Let $\widehat{\theta}$ be the median individual (satisfying $F(\widehat{\theta})=1 / 2$ ). Further, assume $w^{\prime}(\theta)>0, r^{\prime}(\theta)>0$, and that $r(\theta) / w(\theta)$ increases with $\theta$. In words, a higher $\theta$ corresponds to higher levels of both capital and labor incomes, and to a larger share of capital income. Finally, we assume $r(0) / w(0)<\bar{r} / \bar{w}<r(1) / w(1)$ where $\bar{r}$ and $\bar{w}$ denote average capital and labor incomes. ${ }^{5}$

All individuals have identical quasi-linear preferences over a numeraire good (non-polluting) and a polluting good, $y$. The goods are produced by a linear technology subject to constant returns to scale in a competitive environment. Normalize the producer price of $y$ at one. Let $q$ denote the consumer price of $y, I(\theta)$ the disposable income (net of taxes or transfers) and $Y$ the total consumption of $y$ (across all individuals). The indirect utility function of an individual of type $\theta$ is given by:

$$
v(q, I, Y)=a(q)+I(\theta)-\varphi(Y)
$$

with $a^{\prime}(q)<0, \varphi^{\prime}(Y)>0$, and $\varphi^{\prime \prime}(Y)>0$. Total consumption of the polluting good thus creates a negative "atmosphere externality" of $\varphi(Y)$. By Roy's identity, the demand for $y$ is given by

$$
y(q)=-\frac{\partial v}{\partial q}=-a^{\prime}(q) .
$$

Note that $y$ is independent of $\theta$. Aggregate consumption of the polluting good is then equal to

$$
Y=\int_{0}^{1} y(q) f(\theta) d \theta=\bar{y}(q)=y(q)
$$

\footnotetext{
${ }^{5}$ These are defined formally by

$$
\bar{r}=\int_{0}^{1} r(\theta) f(\theta) d \theta \quad \text { and } \quad \bar{w}=\int_{0}^{1} w(\theta) f(\theta) d \theta .
$$
}

This assumption ensures that $r(\theta) / w(\theta)$ will be greater than $\bar{r} / \bar{w}$ for some individuals, and less than it for others. 
so that total, average and individual consumption levels are all equal.

Good $y$ is subject to a "pollution tax" levied at the rate of $(q-1)$ per unit of output. The proceeds of the tax are refunded through reductions in labor and capital income taxes. To simplify notation, we do not explicitly include pre-existing income taxes. This implies that the net of tax income of individual $\theta$ is given by

$$
I(\theta)=\left(1+g_{r}\right) r(\theta)+\left(1+g_{w}\right) w(\theta)
$$

where $g_{r} \geq 0$ and $g_{w} \geq 0$ are the refund rates on capital and wage incomes.

The tax and refund rates are related through the government's budget constraint

$$
R(q) \equiv(q-1) y(q)=g_{r} \bar{r}+g_{w} \bar{w},
$$

where $R(q)$ is tax revenue ${ }^{6}$. Observe that, in light of (5), the government has only two degrees of freedom in choosing its policy instruments. Once $q$ and, say, $g_{r}$ are set, $g_{w}$ is automatically determined. To represent this in a more symmetric way and to characterize the refund system through a single parameter, we introduce the concept of a "refund rule". This specifies the proportion $\alpha$ of tax proceeds that must be refunded on the basis of wage incomes. Formally, $\alpha$ is defined such that

$$
\alpha=\frac{g_{w} \bar{w}}{R(q)}=1-\frac{g_{r} \bar{r}}{R(q)} .
$$

With this notation, the tax-cum-refund policy is completely characterized by the pollution tax (or the consumer price of $y$ ) and by the refund rule; i.e. by the two parameters $q$ and $\alpha$. In the remainder of the paper we study the determination of these two parameters through the political process.

The first obvious candidate to consider is a simultaneous voting procedure. However, it is well-known from the voting literature that conditions for the existence of a Condorcet winner in a two-dimensional setting are

\footnotetext{
${ }^{6}$ Observe that $g_{r} \geq 0$ and $g_{w} \geq 0$ result in $R(q) \geq 0$,i.e. we rule out pollution subsidies.
} 
extremely restrictive. ${ }^{7}$ Consequently, we concentrate on other majority voting procedures to determine the values of $\alpha$ and of $q$. Sections $4-5$ study the sequential vote over these two instruments. Section 6 assumes that voting takes place separately over the two instruments, and looks for a fixed point in this setting. Section 7 considers simultaneous vote over $(q, \alpha)$ when the pairs considered are restricted to those most preferred by at least one voter. Finally, we show by way of examples that the equilibrium obtained with these restrictions may or may not be a Condorcet winner when the restrictions are lifted.

As a first step, we begin by examining a $\theta$-type person's preferences over $(q, \alpha)$. This will be a useful ingredient in the subsequent analysis. In particular, we will characterize the preferred solution of the median endowed individual $\widehat{\theta}$.

\section{Preferences over $(q, \alpha)$ and the median individ- ual's optimum}

A voter's preferences over tax and refund policies can be derived from the individual's utility function (1), by making use of (3)-(6). This yields the following reduced indirect utility function,

$$
V(q, \alpha, \theta)=a(q)+m(\theta)+\delta(\theta, \alpha) R(q)-\varphi(y(q)),
$$

where

$$
\delta(\theta, \alpha) \equiv(1-\alpha) \frac{r(\theta)}{\bar{r}}+\alpha \frac{w(\theta)}{\bar{w}} .
$$

Note that $\delta(\theta, \alpha)$ shows what proportion of his tax payment a person of type $\theta$ gets back in refunds. To see this, substitute for $\alpha$ from (6) into (8). This yields

$$
\delta(\theta, \alpha)=\frac{g_{r} r(\theta)+g_{w} w(\theta)}{R(q)} .
$$

It is clear from (7) that the size of $\delta(\theta, \alpha)$ is a crucial determinant of the impact of $q$ on $V(q, \alpha, \theta)$. Moreover, $\delta(\theta, \alpha)$ is the only direct channel through

\footnotetext{
${ }^{7}$ See e.g., Davis et al. (1972), Plott(1967), Tovey(1992) or Schoffield and Tovey(1992).
} 


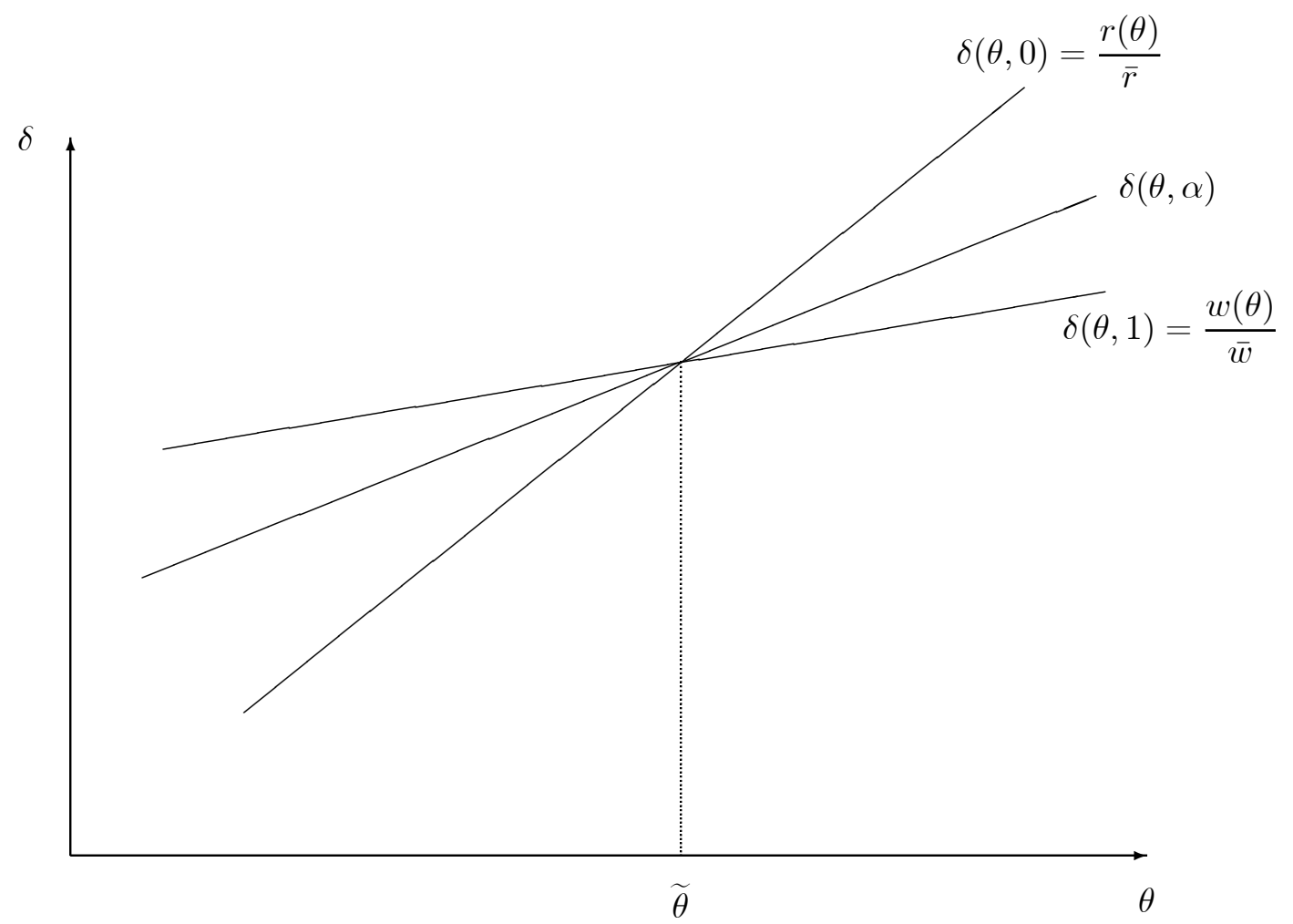

Figure 1: Depicting $\delta(\theta, \alpha)$ for different values of $\alpha$.

which $\alpha$ affects $V(q, \alpha, \theta)$. Lemma 1, from Cremer et al. (2001), also proved in the Appendix, summarizes the properties of $\delta(\theta, \alpha)$ that will be useful in our future discussions. See also Figure 1.

Lemma 1 Let $\delta(\theta, \alpha)$, defined by (8), denote the proportion of the tax payment that a person of type $\theta$ gets back in refunds. We have:

(i) $\delta(\theta, \alpha)$ is an increasing function of $\theta: \delta_{\theta}(\theta, \alpha)>0$.

(ii) $\delta(\theta, \alpha)$ is a linear function of $\alpha$ with the slope

$$
\delta_{\alpha}(\theta)=\frac{w(\theta)}{\bar{w}}-\frac{r(\theta)}{\bar{r}} .
$$

This is positive, zero, or negative depending on the individual's type.

(iii) There exists one, and only one, value of $\theta$ at which $\delta_{\alpha}(\theta)=0$. This value, $\widetilde{\theta}$, denotes the person for whom $\delta(\theta, \alpha)$ is independent of $\alpha$ so that varying $\alpha$ will have no direct impact on his utility, $V(q, \alpha, \theta)$. For the rest 
of the population, $\delta_{\alpha}(\theta) \gtreqless 0 \Leftrightarrow \theta \lesseqgtr \widetilde{\theta}$.

Next, we characterize the most-preferred policy of individual $\theta$, denoted by $\left(q^{*}(\theta), \alpha^{*}(\theta)\right)$. As a first step, we derive $\theta$ 's most-preferred value of $\alpha$ conditional on $q, \alpha^{\dagger}(\theta ; q)$, and his most-preferred tax rate conditional on $\alpha$, $q^{\dagger}(\theta ; \alpha)$. Thus differentiate (7) partially with respect to $\alpha$

$$
\frac{\partial V(q, \alpha, \theta)}{\partial \alpha}=\delta_{\alpha}(\theta) R(q)=\left[\frac{w(\theta)}{\bar{w}}-\frac{r(\theta)}{\bar{r}}\right] R(q) .
$$

$>$ From Lemma $1, \delta_{\alpha}(\theta) \gtreqless 0 \Leftrightarrow \theta \lesseqgtr \widetilde{\theta}$. Consequently, equation (11) implies that an individual's most-preferred level of $\alpha$ is independent of $q$. It will be either one or zero, depending on whether $\theta<\tilde{\theta}$ or $\theta>\widetilde{\theta}$.

Turning to the most-preferred tax rate, differentiate (7) partially with respect to $q$,

$$
\frac{\partial V(q, \alpha, \theta)}{\partial q}=[\delta(\theta, \alpha)-1] R^{\prime}(q)-\left[\varphi^{\prime}-(q-1)\right] y^{\prime}(q),
$$

where

$$
R^{\prime}(q)=y(q)+(q-1) y^{\prime}(q)
$$

is the marginal tax revenue. Assuming an interior maximum, one can then determine $q^{\dagger}(\theta ; \alpha)$ by setting equation (12) equal to zero. ${ }^{8}$ There are two interesting aspects to this solution. One is that $q^{\dagger}(\theta ; \alpha)$ is monotonically increasing in $\theta$. The other is that $q^{\dagger}(\theta ; \alpha)$ is monotonically increasing in $\alpha$, if $\theta<\widetilde{\theta}$ and decreasing if $\theta>\widetilde{\theta}$. Specifically, we have:

Lemma 2 Assume $q^{\dagger}(\theta ; \alpha)$ is an interior maximum and $R^{\prime}\left(q^{\dagger}(\theta ; \alpha)\right)>0 .{ }^{9}$

Then,

\footnotetext{
${ }^{8} \mathrm{~A}$ set of sufficient conditions for this is that $\partial V(1, \alpha, \theta) / \partial q>0$ and $\partial^{2} V\left[q^{\dagger}(\theta ; \alpha), \alpha, \theta\right) / \partial q^{2}<0$. In turn, the latter condition will be satisfied if

$$
\left(2 \delta-1-\varphi^{\prime \prime} y^{\prime}\right) y^{\prime 2}-(\delta-1) y y^{\prime \prime}>0,
$$

at $q=q^{\dagger}(\theta ; \alpha)$.

${ }^{9}$ As pointed out by Cremer et al. (2001), this assumption is necessarily satisfied in the absence of a negative externality. In that case, no one would vote for a tax rate that is on the downward section of the Laffer curve; see, Guesnerie and Jerison (1991).
} 
(i) $\partial q^{\dagger}(\theta ; \alpha) / \partial \theta$ has the same sign as $\delta_{\theta}(\theta, \alpha)$, so that $q^{\dagger}(\theta ; \alpha)$ is monotonically increasing in $\theta$.

(ii) $\partial q^{\dagger}(\theta ; \alpha) / \partial \alpha$ has the same sign as $\delta_{\alpha}(\theta)$, so that

$$
\frac{\partial q^{\dagger}(\theta ; \alpha)}{\partial \alpha} \gtreqless 0 \Leftrightarrow \theta \lesseqgtr \widetilde{\theta}
$$

Proof. With $q^{\dagger}(\theta ; \alpha)$ being the interior maximum of $V(q, \alpha, \theta)$, we have

$$
\begin{aligned}
\frac{\partial V\left(q^{\dagger}(\theta ; \alpha), \alpha, \theta\right)}{\partial q} & \equiv 0 \\
\frac{\partial^{2} V\left(q^{\dagger}(\theta ; \alpha), \alpha, \theta\right)}{\partial q^{2}} & <0
\end{aligned}
$$

Now differentiating (14) partially with respect to $\theta$ yields

$$
\frac{\partial^{2} V\left(q^{\dagger}(\theta ; \alpha), \alpha, \theta\right)}{\partial q^{2}} \frac{\partial q^{\dagger}(\theta ; \alpha)}{\partial \theta}+\frac{\partial^{2} V\left(q^{\dagger}(\theta ; \alpha), \alpha, \theta\right)}{\partial \theta \partial q}=0 .
$$

Thus, from (15), $\partial q^{\dagger}(\theta ; \alpha) / \partial \theta$ has the same sign as $\partial^{2} V\left(q^{\dagger}(\theta ; \alpha), \alpha, \theta\right) / \partial \theta \partial q$. This latter expression in turn has the same sign as $\delta_{\theta}(\theta, \alpha)$. To see this, differentiate (12) partially with respect to $\theta$. We have

$$
\frac{\partial^{2} V(q, \alpha, \theta)}{\partial \theta \partial q}=\delta_{\theta}(\theta, \alpha) R^{\prime}(q)
$$

with $R^{\prime}\left(q^{\dagger}(\theta ; \alpha)\right)>0$. Finally, the stated property of $\delta_{\theta}(\theta, \alpha)$ in Lemma 1 (i), completes the proof of part (i).

Next, differentiating (14) partially with respect to $\alpha$ yields

$$
\frac{\partial^{2} V\left(q^{\dagger}(\theta ; \alpha), \alpha, \theta\right)}{\partial q^{2}} \frac{\partial q^{\dagger}(\theta ; \alpha)}{\partial \alpha}+\frac{\partial^{2} V\left(q^{\dagger}(\theta ; \alpha), \alpha, \theta\right)}{\partial \alpha \partial q}=0 .
$$

Again, from (15), $\partial q^{\dagger}(\theta ; \alpha) / \partial \alpha$ has the same sign as $\partial^{2} V\left(q^{\dagger}(\theta ; \alpha), \alpha, \theta\right) / \partial \alpha \partial q$. In turn, this latter expression has the same sign as $\delta_{\alpha}(\theta, \alpha)$. To prove this, differentiate (12) partially with respect to $\alpha$. We have

$$
\frac{\partial^{2} V(q, \alpha, \theta)}{\partial \alpha \partial q}=\delta_{\alpha}(\theta) R^{\prime}(q)
$$

with $R^{\prime}\left(q^{\dagger}(\theta ; \alpha)\right)>0$. As with part $(i)$, the stated property of $\delta_{\alpha}(\theta, \alpha)$ in Lemma 1 (iii), completes the proof of part (ii). 
We are now in a position to characterize $\left(q^{*}(\theta), \alpha^{*}(\theta)\right)$, the most-preferred policy of individual $\theta$. First, because $\alpha^{\dagger}(\theta ; q)$ is independent of $q, \alpha^{*}(\theta)=$ $\alpha^{\dagger}(\theta ; q)$. Hence $\alpha^{*}(\theta)$ has the same characterization as $\alpha^{\dagger}(\theta ; q)$. Turning to $q^{*}(\theta)$, we prove below that like $q^{\dagger}(\theta ; \alpha), q^{*}(\theta)$ is monotonically increasing in $\theta$.

Proposition 1 (i) $\alpha^{\dagger}(\theta ; q)$ is decreasing in $\theta$ and given by

$$
\alpha^{\dagger}(\theta ; q)=\alpha^{*}(\theta)= \begin{cases}1 & \text { if } \theta<\widetilde{\theta} \\ 0 & \text { if } \theta>\widetilde{\theta} \\ \in[0,1] & \text { if } \theta=\tilde{\theta}\end{cases}
$$

(ii) Assume $q^{\dagger}(\theta ; \alpha)$ is an interior maximum, and $R^{\prime}\left(q^{\dagger}(\theta ; \alpha)\right)>0$. Then, $q^{*}(\theta)=q^{\dagger}\left(\theta ; \alpha^{*}(\theta)\right)$ is monotonically increasing in $\theta$.

Proof. Part (i) follows from equation (11) and Lemma 1. To prove part (ii), consider all $\theta \neq \widetilde{\theta} \in[0,1]$. Now from $(18), \alpha^{*}(\theta)=1$ on $[0, \widetilde{\theta}[$ and $\alpha^{*}(\theta)=0$ on $\left.] \widetilde{\theta}, 1\right]$. We also know from Lemma 2 (iii) that $q^{\dagger}(\theta ; \alpha)$ is increasing in $\theta$ for any given value of $\alpha$. Consequently, over the intervals $[0, \widetilde{\theta}[$ and $] \widetilde{\theta}, 1], q^{*}(\theta)=q^{\dagger}\left(\theta ; \alpha^{*}(\theta)\right)$ is increasing in $\theta$.

It remains to show that $q^{*}(\theta)=q^{\dagger}\left(\theta ; \alpha^{*}(\theta)\right)$ is continuous at $\widetilde{\theta}$. With $q^{\dagger}(\theta ; \alpha)$ being continuous in $\theta$, it will suffice to show that $q^{\dagger}(\widetilde{\theta} ; \alpha)$ is independent of $\alpha$. This we have already shown in (13). See Figure 2.

We close this section by characterizing the most-preferred policy of the median individual. As we shall see, the median individual turns out to be the decisive voter in all the voting procedures we study with two possible exceptions.

Proposition 2 Let $(\widehat{q}, \widehat{\alpha})=\left(q^{*}(\widehat{\theta}), \alpha^{*}(\widehat{\theta})\right)$ denote the most-preferred taxcum-refund policy of the median individual. We have:

(i)

$$
\widehat{\alpha}= \begin{cases}1 & \text { if } \quad \widehat{\theta}<\tilde{\theta} \\ 0 & \text { if } \quad \widehat{\theta}>\widetilde{\theta} \\ \in[0,1] & \text { if } \quad \widehat{\theta}=\widetilde{\theta}\end{cases}
$$




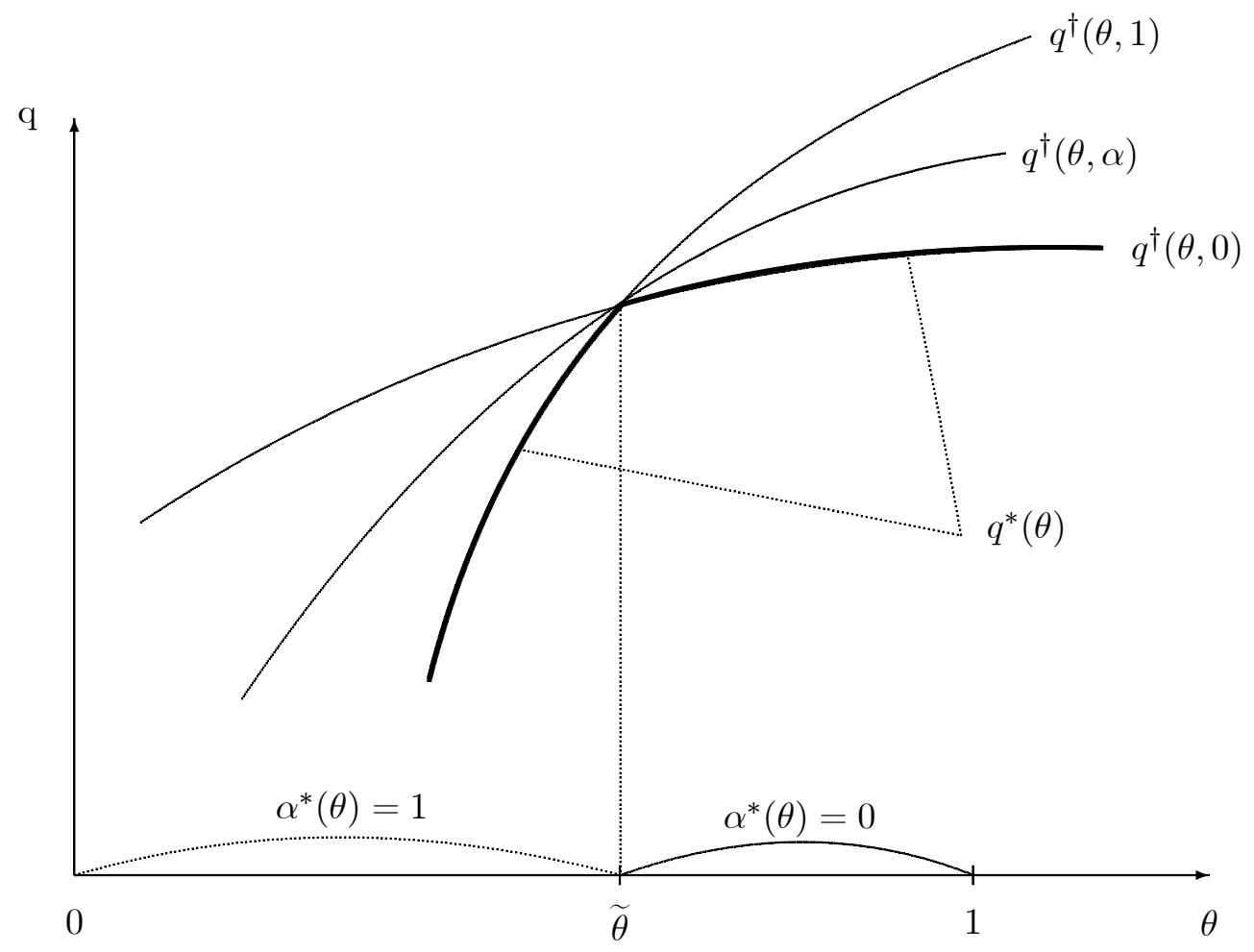

Figure 2: Determination of $q^{*}(\theta)$. 
(ii)

$$
\widehat{q} \gtreqless q^{F} \Leftrightarrow \max \left[\frac{\widehat{w}}{\bar{w}}, \frac{\widehat{r}}{\bar{r}}\right] \gtreqless 1,
$$

where $\left(q^{F}-1\right)=\varphi^{\prime}$ is the first-best Pigouvian tax.

The proof of part (i) follows as an application of Proposition 1 (i) to the median individual. Part (ii) is proved in the Appendix. This establishes that the median individual's most-preferred tax rate may exceed the Pigouvian rate or fall short of it depending on the relationship between median and mean incomes (wage and capital). To understand the intuition behind this result, assume first that $1>\widehat{w} / \bar{w}>\widehat{r} / \bar{r}$. That is, assume that the median is smaller than the mean for both sources of income and that the ratio of median to mean is smaller for capital than for wage income distribution. The latter assumption is one indicator of the fact that capital income distribution is "more skewed" than the wage income distribution. Under this assumption, the median will want to have the entire tax refunds based on labor income; consequently, we have $\widehat{\alpha}=1$. However, with $1>\widehat{w} / \bar{w}$, even the mostpreferred level of $\alpha$ is not "good enough" to ensure the median individual a full refund of his tax payments. ${ }^{10}$ Consequently, he favors a tax which is less than the (socially) optimal (Pigouvian) level.

Under the alternative assumption that $\widehat{w} / \bar{w}>1>\widehat{r} / \bar{r}$, we again obtain $\widehat{\alpha}=1$, but this now yields a more than full refund for the median individual. Consequently, he favors a tax which exceeds the Pigouvian level. Finally, when $\widehat{r} / \bar{r}>\widehat{w} / \bar{w}$, we will have $\widehat{\alpha}=0$; the median now wants all refunds to be based on capital income. The remaining part of the argument then goes through exactly as in the previous cases. ${ }^{11}$

This discussion, with its normative flavor is somewhat orthogonal to the main focus of the paper. Nevertheless, the arguments are quite telling for they highlight the implications of the nonseparability between efficiency and equity brought about by the political process.

\footnotetext{
${ }^{10}$ Formally, $\delta(\widehat{\theta}, \widehat{\alpha})=\delta(\widehat{\theta}, 1)<1$.

${ }^{11}$ This result generalizes a proposition in Cremer et al. (2002).
} 


\section{Sequential vote: $\alpha$ then $q$}

Consider a sequential voting procedure of the following sort. In the second stage, $q$ is determined by majority voting for a given level of $\alpha$; denote the voting equilibrium by $q^{E}(\alpha)$. In the first stage, individuals vote on $\alpha$, anticipating the induced voting equilibrium in the second stage. ${ }^{12}$ To determine the equilibrium of this sequential vote, we proceed by backward induction.

\subsection{Stage 2: voting on $q$ given $\alpha$}

The following Lemma from Cremer et al. (2001), also proved in the Appendix, establishes the existence of a majority voting equilibrium for $q$ conditional on $\alpha$, denoted by $q^{E}(\alpha)$.

Lemma 3 For any $\alpha \in[0,1]$, there exists a majority voting equilibrium level of $q: q^{E}(\alpha)$. This is given by the most-preferred choice of the median individual; that is,

$$
q^{E}(\alpha)=q^{\dagger}(\widehat{\theta} ; \alpha), \quad \forall \alpha \in[0,1]
$$

\subsection{Stage 1: voting on $\alpha$}

In the first stage, voter $\theta$ has preferences over $\alpha$ as described by

$$
V^{I}(\alpha, \theta)=V\left(q^{E}(\alpha), \alpha, \theta\right)
$$

where $V$ is defined in (7). Let

$$
\alpha^{I}(\theta)=\arg \max _{\alpha \in[0,1]}\left[V^{I}(\alpha, \theta)\right]
$$

denote the most preferred level of $\alpha$ for voter $\theta$. One has to study the properties of $\alpha^{I}(\theta)$ to determine if a voting equilibrium exists.

\footnotetext{
${ }^{12}$ When defining the equilibrium in the sequential voting procedure, we assume that the second-stage equilibrium exists and is unique. This is the case in our setting as will become clear below.
} 


\subsubsection{Preferences over $\alpha$ in the first stage}

Differentiate (22) totally with respect to $\alpha$. This yields

$$
\frac{\partial V^{I}(\alpha, \theta)}{\partial \alpha}=\underbrace{\frac{\partial V\left(q^{E}(\alpha), \alpha, \theta\right)}{\partial \alpha}}_{A}+\underbrace{\frac{\partial V\left(q^{E}(\alpha), \alpha, \theta\right)}{\partial q} \frac{d q^{E}(\alpha)}{d \alpha}}_{B} .
$$

A variation in $\alpha$ has two effects on an individual's utility: a direct effect, $A$, and an indirect effect, $B$, associated with the induced variation in the second stage voting equilibrium value of $q^{E}$. Consider the two terms separately.

Direct effect: A This is specified by (11) and we have $A>0$ for $\theta<\widetilde{\theta}$, and $A<0$ for $\theta>\widetilde{\theta}$. According to the direct effect, then, all individuals in the interval $\theta<\widetilde{\theta}$ favor $\alpha=1$, while those in $\theta>\widetilde{\theta}$ favor $\alpha=0$. See Figure 3.

Indirect effect: B The analysis of this term is more complex. First, we know from (16) that $\partial^{2} V\left(q^{E}(\alpha), \alpha, \theta\right) / \partial \theta \partial q>0$, if $R^{\prime}\left(q^{E}\right)>0$. That is, $\partial V\left(q^{E}(\alpha), \alpha, \theta\right) / \partial q$ is increasing in $\theta$. Now with $q^{E}(\alpha)=q^{\dagger}(\widehat{\theta} ; \alpha)$ being the second stage median voter's most-preferred value of $q$, we have $\partial V\left(q^{E}(\alpha), \alpha, \widehat{\theta}\right) / \partial q=0$. These properties imply that

$$
\frac{\partial V\left(q^{E}(\alpha), \alpha, \theta\right)}{\partial q} \lesseqgtr 0 \quad \Leftrightarrow \quad \theta \lesseqgtr \widehat{\theta} .
$$

Second, with $q^{E}(\alpha)=q^{\dagger}(\widehat{\theta} ; \alpha)$, we have from Lemma (2) (ii) that

$$
\frac{d q^{E}(\alpha)}{d \alpha} \gtreqless 0 \quad \Leftrightarrow \quad \widehat{\theta} \lesseqgtr \widetilde{\theta} .
$$

That is, thee second-stage voting equilibrium of $q$ is increasing or decreasing in $\alpha$ depending on whether $\widehat{\theta}<\widetilde{\theta}$ or $\widehat{\theta}>\widetilde{\theta}$. In the former case, the sign of the indirect effect is as specified by (24). In the latter case, the sign is reversed. See Figure 3. 


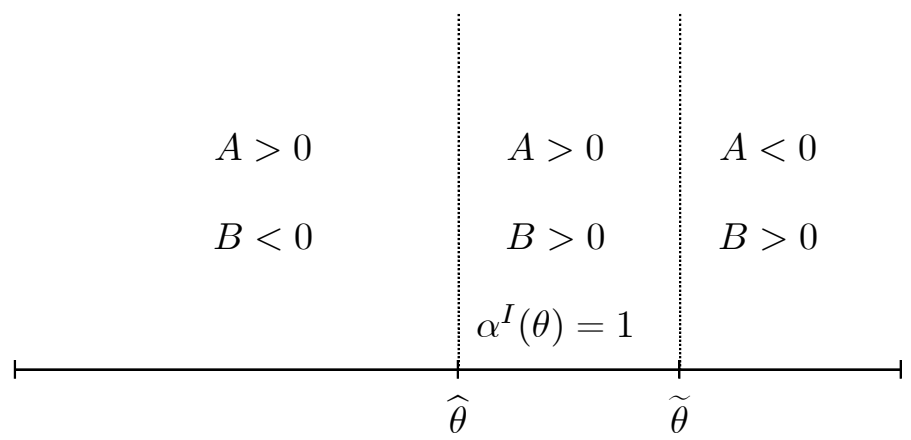

$3 \mathrm{a}: \frac{\widetilde{w}}{\widehat{w}}>\frac{\widetilde{r}}{\widehat{r}} \quad(\widehat{\theta}<\widetilde{\theta})$

\begin{tabular}{|c|c|c|}
\hline$A>0$ & $A<0$ & $A<0$ \\
\hline$B>0$ & $B>0$ & $B<0$ \\
\hline$\alpha^{I}(\theta)=1$ & & $\alpha^{I}(\theta)=0$ \\
\hline
\end{tabular}

$3 \mathrm{~b}: \frac{\widetilde{w}}{\widehat{w}}<\frac{\widetilde{r}}{\widehat{r}} \quad(\widehat{\theta}>\widetilde{\theta})$

Figure 3: Determination of $\alpha^{I}(\theta)$. 
Total effect When $A$ and $B$ are of the same sign, an individual's most preferred value for $\alpha$ is a corner solution. Specifically, $A>0$ and $B>0$ implies $\alpha^{I}(\theta)=1$, while $A<0$ and $B<0$ yields $\alpha^{I}(\theta)=0$. When $A$ and $B$ are of opposite signs, no clearcut conclusion can be drawn. The individual's most preferred $\alpha$ may, but need not, be interior. ${ }^{13}$ Observe that for the median individual only the first term is relevant as $B=0$. He will then always choose a corner solution except if $\widehat{\theta}=\widetilde{\theta}$, in which case he is indifferent to any value of $\alpha \in[0,1]$.

\subsubsection{The decisive voter and the median individual}

If $A$ were the only effect, the decisive voter would necessarily be $\widehat{\theta}$. When both effects are present, one must distinguish between two cases as illustrated in Figure 3. In case (b), $\widehat{\theta}>\widetilde{\theta}$ : The median individual and all individuals to his right have the same most-preferred value for $\alpha$; namely $\alpha=0$. By definition, then, they form a majority and the median individual is decisive. In case (a), $\widehat{\theta}<\widetilde{\theta}$, and it is not a priori clear whether the median individual is decisive or not. This is because direct and indirect effects are now of conflicting signs at both ends of the distribution; see Section 9 below for numerical examples where the median is and is not decisive. Observe that when the median is not decisive we can have two types of situations. First, we can have an equilibrium with a different pivotal voter. Second, and more seriously, the equilibrium may fail to exist altogether. ${ }^{14}$

The following proposition summarizes the main results of this section.

Proposition 3 Assume that the policy $(q, \alpha)$ is determined through a sequential majority voting procedure. The second stage vote is on $q$ for a given value of $\alpha$. In the first stage, individuals vote on $\alpha$ anticipating the induced

\footnotetext{
${ }^{13}$ While the signs of $A$ and $B$ do not depend on $\alpha$, their absolute values are affected by $\alpha$. This makes an interior solution possible.

${ }^{14}$ We have run numerical simulations (along the lines of those presented in Section 9) which show that both type of regimes can effectively occur. However, the distinction between these cases is not essential for the remainder of the paper. Consequently, we shall not discuss this in detail here.
} 
second-stage equilibrium. If $\widehat{\theta}>\widetilde{\theta}$, the voting equilibrium exists and is given by the most-preferred policy of the median individual, $(\widehat{q}, \widehat{\alpha})=\left(q^{*}(\widehat{\theta}), \alpha^{*}(\widehat{\theta})\right)$, as characterized in Proposition 2. On the other hand, if $\widehat{\theta}<\widetilde{\theta}$, the median individual may or may not be decisive.

\section{Sequential vote: $q$ then $\alpha$}

Consider now an alternative sequential procedure in which $q$ is determined in the first stage and $\alpha$ in the second. That is, reverse the sequence considered in Section 4. As in that section, we solve this game by backward induction.

\subsection{Stage 2: voting on $\alpha$ given $q$}

To determine individual $\theta$ 's most-preferred level of $\alpha$, for any given level of $q$, it is sufficient to use the properties of $V(q, \alpha, \theta)$ established in Section 3. First, recall that $\partial V / \partial \alpha$ is given by (11). Consequently $V$ is linear in $\alpha$ which implies that the voting equilibrium for $\alpha$ exists. Furthermore expression (11), together with Lemma 1 (ii), implies that the most-preferred level of $\alpha$ is a corner solution and that it is independent of $q$. This is given by $\alpha^{*}(\theta)$ as defined by (19). Finally, the property that $\alpha^{*}(\theta)$ is a monotonic (decreasing) function of $\theta$ implies that the median individual is the decisive voter so that

$$
\alpha^{E}(q)=\alpha^{*}(\widehat{\theta})=\widehat{\alpha} \quad \forall q,
$$

where $\alpha^{E}(q)$ denotes the second-stage voting equilibrium, and $\widehat{\alpha}$ is defined by (19).

\subsection{Stage 1: voting on $q$}

When voting over $q$, individuals anticipate that the second-stage equilibrium will be $\alpha^{*}(\widehat{\theta})$, so that their first-stage utility is

$$
V^{I}(q, \theta)=V\left(q, \alpha^{*}(\widehat{\theta}), \theta\right)
$$


Given that $\alpha^{*}(\widehat{\theta})$ is independent of $q$, one can apply Lemma 3 to show that the median individual is also decisive in the first stage:

$$
q^{E}(\widehat{\alpha})=q^{\dagger}(\widehat{\theta} ; \widehat{\alpha})=q^{*}(\widehat{\theta})=\widehat{q}
$$

Anticipating that his preferred $\alpha$ will prevail in the second stage, the median individual will simply choose $q^{*}(\widehat{\theta})=\widehat{q}$. This is the level of $q$ that he would choose, if he could directly set the entire policy $(q, \alpha)$.

The following proposition summarizes the results obtained in this section.

Proposition 4 Assume that the policy $(q, \alpha)$ is determined through a sequential majority voting procedure. The second-stage vote is on $\alpha$ for a given value of $q$. In the first stage, individuals vote on $q$ anticipating the induced second-stage equilibrium. The voting equilibrium exists and is given by the most-preferred policy of the median individual, $(\widehat{q}, \widehat{\alpha})=\left(q^{*}(\widehat{\theta}), \alpha^{*}(\widehat{\theta})\right)$, characterized in Proposition 2.

\section{Shepsle's procedure}

This section considers the choice procedure proposed by Shepsle (1979) where one assumes that the two votes (over $\alpha$ and $q$ ) are taken separately. This would be the case if, for instance, each aspect of the problem (the value of the tax rate and the structure of the refunds) were chosen by a different committee. Because preferences over each variable depend on the value assumed by the other, one must first compute the majority voting reaction functions $q^{E}(\alpha)$ and $\alpha^{E}(q)$. A Shepsle equilibrium is then a pair $\left(q^{S}, \alpha^{S}\right)$ such that each variable in this pair is the majority voting equilibrium conditional on the value of the other variable, i.e. $q^{S}=q^{E}\left(\alpha^{S}\right)$ and $\alpha^{S}=\alpha^{E}\left(q^{S}\right)$.

The determination of the Shepsle equilibrium follows directly from the results obtained for the second-stage voting equilibria of Sections 4-5. First, 
observe that equation (26) implies $\alpha^{S}=\widehat{\alpha}$. With the majority voting equilibrium value of $\alpha$ (for a given $q$ ) being independent of $q$, the Shepsle level of $\alpha$ is also independent of $q$. Evaluating (21) at $\alpha^{S}=\widehat{\alpha}$ then yields,

$$
q^{S}=q^{E}(\widehat{\alpha})=q^{\dagger}(\widehat{\theta} ; \widehat{\alpha})=q^{*}(\widehat{\theta})=\widehat{q}
$$

This establishes the following result.

Proposition 5 Assume that the policy $(q, \alpha)$ is determined through a Shepsle procedure where the equilibrium policy is a pair $\left(q^{S}, \alpha^{S}\right)$ such that $q^{S}=$ $q^{E}\left(\alpha^{S}\right)$ and $\alpha^{S}=\alpha^{E}\left(q^{S}\right)$ (i.e., $q^{S}$ is the voting equilibrium given $\alpha^{S}$, and $\alpha^{S}$ is the voting equilibrium given $\left.q^{S}\right)$. The equilibrium of this procedure exists and is given by the most-preferred policy of the median individual, i.e.,

$$
\left(q^{S}, \alpha^{S}\right)=(\widehat{q}, \widehat{\alpha})
$$

where $(\widehat{q}, \widehat{\alpha})$ is characterized in Proposition 2.

\section{$7 \quad$ Voting over blisspoints}

We now consider a procedure where the set of admissible alternatives is restricted to the individual blisspoints $\left(q^{*}(\theta), \alpha^{*}(\theta)\right)$. Such a restriction is justified, for instance, in the context of a representative democracy where "the candidates" cannot commit to a policy. Consequently, voters anticipate that each candidate will implement his own most-preferred policy if elected. This assumption has been used for instance by Osborne-Slivinski (1996) and Besley-Coate(1997) in the so-called citizen-candidate model. Unlike these papers, however, we do not model the initial stage of the game wherein citizens decide if they want to be a candidate. Instead, we effectively assume that all citizens are potential candidates. Note that, as long as the median individual is a candidate, our results remain intact. And the candidacy of the median individual appears very likely because he will be decisive if he stands. 
To study the voting equilibrium of such a procedure, we first have to look at the properties of the set of individual blisspoints. We have shown in Section 3, Proposition 1 , that $\alpha^{*}(\theta)$ is decreasing in $\theta$ and given by (18), and $q^{*}(\theta)$ is monotonically increasing in $\theta$. We now derive the voting equilibrium.

Proposition 6 Assume that the policy $(q, \alpha)$ is determined through a majority voting procedure in which the set of admissible alternatives is restricted to the individual blisspoints $\left(q^{*}(\theta), \alpha^{*}(\theta)\right)$, and that $V(q, \alpha ; \theta)$ is single-peaked in $q$ for all values of $\alpha$ and $\theta$. The equilibrium of this procedure exists and is given by the most-preferred policy of the median individual, $(\widehat{q}, \widehat{\alpha})$, as characterized in Proposition 2.

Proof. Consider $V\left(q^{*}\left(\theta^{\prime}\right), \alpha^{*}\left(\theta^{\prime}\right) ; \theta\right)$ : the preferences of a $\theta$-type person $\in[0,1]$ over the blisspoints $\left(q^{*}\left(\theta^{\prime}\right), \alpha^{*}\left(\theta^{\prime}\right)\right)$ of $\theta^{\prime} \in[0,1]$. We prove that these preferences are single-peaked. We can then apply the median voter theorem which states that the majority voting equilibrium is the point most preferred by the median individual.

We have

$$
V\left(q^{*}\left(\theta^{\prime}\right), \alpha^{*}\left(\theta^{\prime}\right) ; \theta\right)= \begin{cases}V\left(q^{*}\left(\theta^{\prime}\right), 1 ; \theta\right) & \forall \theta^{\prime}<\tilde{\theta} \\ V\left(q^{*}\left(\theta^{\prime}\right), \alpha ; \theta\right) & \theta^{\prime}=\widetilde{\theta} \\ V\left(q^{*}\left(\theta^{\prime}\right), 0 ; \theta\right) & \forall \theta^{\prime}>\widetilde{\theta}\end{cases}
$$

Assume first that $\theta<\widetilde{\theta}$. From (11), $\partial V(q, \alpha, \theta) / \partial \alpha>0$ indicating that $V\left(q^{*}\left(\theta^{\prime}\right), 1 ; \theta\right)$ lies above $V\left(q^{*}\left(\theta^{\prime}\right), 0 ; \theta\right)$ for all values of $q$. Moreover, from Lemma (2) (ii), we have that over this interval, $\partial q^{\dagger}(\theta ; \alpha) / \partial \alpha>0$. Hence the peak in $V\left(q^{*}\left(\theta^{\prime}\right), 1 ; \theta\right)$ is to the right of the peak in $V\left(q^{*}\left(\theta^{\prime}\right), 0 ; \theta\right)$. Now we also have, from Lemma (2) (i), that $\partial q^{\dagger}(\theta ; \alpha) / \partial \theta>0$ for all $\alpha$. Consequently, the peak in $V\left(q^{*}\left(\theta^{\prime}\right), 1 ; \theta\right)$ is to the left of $q^{*}(\widetilde{\theta})$. We thus obtain the curves $V\left(q^{*}\left(\theta^{\prime}\right), 1 ; \theta\right)$, the utility attained for the blisspoints $\left\{\alpha^{*}\left(\theta^{\prime}\right)=1, q^{*}\left(\theta^{\prime}\right)<\right.$ $\left.q^{*}(\widetilde{\theta})\right\}$, and $V\left(q^{*}\left(\theta^{\prime}\right), 0 ; \theta\right)$, the utility attained for the blisspoints $\left\{\alpha^{*}\left(\theta^{\prime}\right)=\right.$ $\left.0, q^{*}\left(\theta^{\prime}\right)>q^{*}(\widetilde{\theta})\right\}$, as shown in Figure 4a (given the single-peakedness of the two curves). The utility of $\theta<\tilde{\theta}$ on the set of blisspoints is single peaked. 
Figure $4 \mathrm{~b}$ shows the same two curves for an individual $\theta>\widetilde{\theta}$. Note that in this case, $\partial V(q, \alpha, \theta) / \partial \alpha<0, \partial q^{\dagger}(\theta ; \alpha) / \partial \alpha<0$ and $\partial q^{\dagger}(\theta ; \alpha) / \partial \theta>0$ assure that the peak of $V\left(q^{*}\left(\theta^{\prime}\right), 0 ; \theta\right)$ is to the right of the peak of $V\left(q^{*}\left(\theta^{\prime}\right), 1 ; \theta\right)$, itself to the right of $q^{*}(\widetilde{\theta})$. This implies the single-peakedness for $\theta>\widetilde{\theta}$. Finally, if $\theta=\widetilde{\theta},(11)$ shows that $V\left(q^{*}\left(\theta^{\prime}\right), \alpha^{*}\left(\theta^{\prime}\right) ; \widetilde{\theta}\right)$ is independent of the value of $\alpha$. Consequently, we will have single-peakedness on the set of blisspoints for $\theta=\widetilde{\theta}$ as well.

The intuition for this proof is as follows. The set of blisspoints can be split into two subsets: one containing the blisspoints with $\alpha=0$, and the other with $\alpha=1$. By assumption, each individual's utility is single-peaked on the subset of blisspoints which corresponds to his preferred value of $\alpha$ ( 1 if $\theta<\widetilde{\theta}, 0$ if $\theta>\widetilde{\theta}$ ). We then have to check that the individual's utility function does not have a second peak on the other subset of blisspoints. It is easy to see that a shift toward the less preferred value of $\alpha$ decreases utility. Furthermore, the proof of Proposition 6 shows that, moving away from $q^{*}(\widetilde{\theta})$ on the least preferred subset of blisspoints always strictly decreases utility.

\section{Unrestricted simultaneous vote}

The simultaneous voting setting we have considered so far assumes that the voters' choices are restricted. In the Shepsle case, only deviations in a single dimension are considered. In the citizen-candidate setting, alternatives are restricted to the set of individual blisspoints. These restrictions increase the likelihood that an equilibrium exists because some deviations are ruled out. It is well known that in an unrestricted simultaneous vote, a Condorcet winner generally fails to exist. However, the results pertaining to the generic nonexistence of a Condorcet winner typically rely on the fact the feasible set is an open set. ${ }^{15}$ In our case, we do not have an open set and blisspoints

\footnotetext{
${ }^{15}$ In this case, first studied by Davis et al. (1972) and Plott (1967), a Condorcet winner exists only if, given an starting point, it is possible to pair all voters in such a way that the members of every pair want to go in opposite directions from one another. If it is impossible to pair all individuals in this manner, there exists a direction that is favored
} 


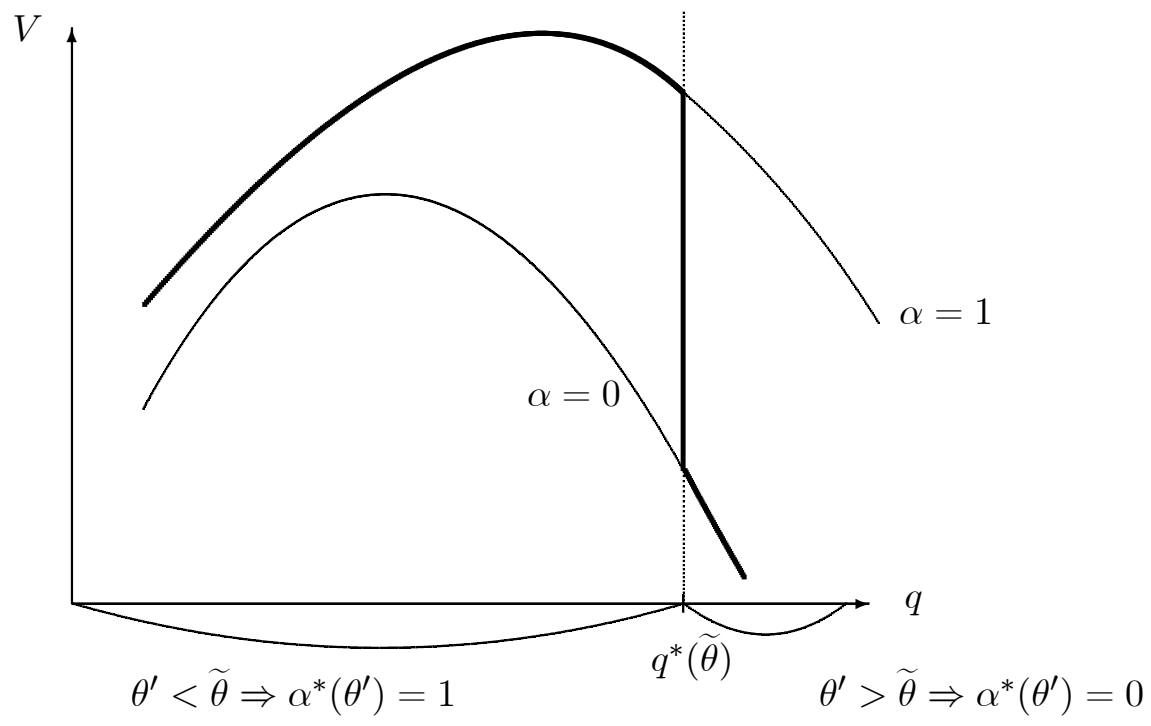

4a: $\theta<\widetilde{\theta}$

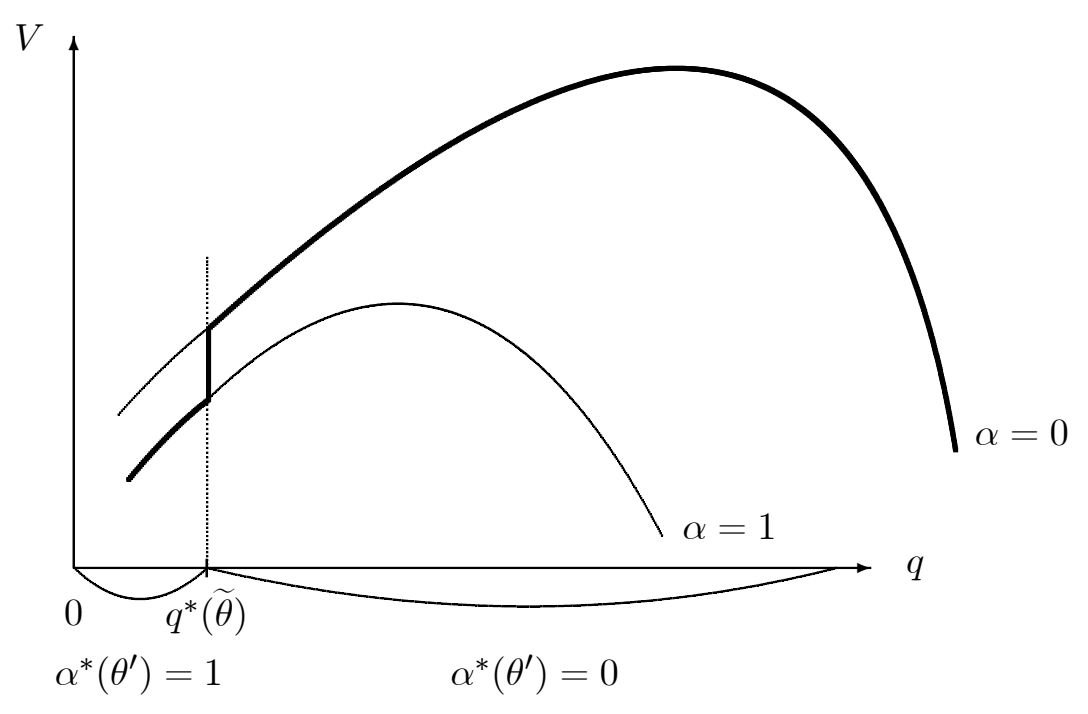

4b: $\theta>\widetilde{\theta}$

Figure 4: The $\theta$ type's preferences over the blisspoints of $\theta^{\prime}$ : $V\left(q^{*}\left(\theta^{\prime}\right), \alpha^{*}\left(\theta^{\prime}\right), \theta\right)$. 
are located on the frontier of the set (corner solution for $\alpha$ ). Consequently, existence of a Condorcet winner cannot be ruled out a priori, at least not on the basis of the usual argument.

The following property, proved in the appendix, is helpful for identifying a candidate Condorcet equilibrium:

Proposition 7 If $(q, \alpha)$ is a Condorcet winner in the unrestricted simultaneous voting game over $q>1$ and $\alpha \in[0,1]$, it is also an equilibrium of the sequential voting games ( $\alpha$ then $q$, and $q$ then $\alpha$ ) and of the Shepsle procedure.

The proof of Proposition 7 is based on the observation that both sequential and the Shepsle procedures impose additional constraints on the set of feasible options. If a pair is a Condorcet winner in the unrestricted simultaneous voting game, it remains a Condorcet winner (and thus a majority voting equilibrium) when the set of feasible options is reduced (provided that it still includes the unrestricted Condorcet winner). Observe that a Condorcet winner is not necessarily the winner in the citizen-candidate model, because the Condorcet winner is not necessarily in the set of individual blisspoints. However, if the Condorcet winner is in the set of individual blisspoints, then it is also the winner in the citizen-candidate setting.

Proposition 7 implies that a Condorcet winner can exist only if the considered alternative procedures all have the same equilibrium. ${ }^{16}$ Based on this proposition, we can distinguish between two cases. One in which the median individual is not decisive in the $\alpha$ then $q$ sequential vote and the other when he is. Under the former, no Condorcet winner exists. In the latter case, on the other hand, there is a possibility of the existence of a Condorcet winner with the candidate being the blisspoint of the median

by a majority of voters, and the considered point is not a Condorcet winner. Existence of a Condorcet winner located on the boundary of a closed feasible set is easier to obtain, because a feasible direction preferred by a majority of individuals would be less likely.

${ }^{16}$ As long as each procedure has a unique equilibrium. 
individual (characterized by Proposition 2). ${ }^{17}$ We emphasize that this candidate equilibrium may, but need not, be a Condorcet winner. All we can say, at this level of generality, is that there is no obvious way to eliminate the candidate equilibrium. To demonstrate that different cases can arise, and to develop further understanding of the pertinent factors, we now turn to the study of some illustrative examples.

\section{Illustrations}

This subsection develops a number of simple numerical examples that satisfy all the assumptions imposed on our general model. They illustrate various possibilities regarding the equilibrium in the sequential $\alpha$ then $q$ vote (median voter decisive or not) and the existence of a Condorcet winner. All examples are based on the following specification

$$
\begin{aligned}
& a(q)=-1-\ln q, \quad \varphi(Y)=Y^{2}, \\
& w(\theta)=\theta^{2}, \quad r(\theta)=\theta^{5} .
\end{aligned}
$$

What differentiates the examples is the distribution of types which is parameterized by $\varepsilon \in[1 / 6,1 / 2[$ so that

$$
f(\theta)= \begin{cases}\frac{2}{3-6 \varepsilon} & \text { if } \theta \leq 1 / 2-\varepsilon \\ \frac{1}{6 \varepsilon_{2}} & \text { if } 1 / 2-\varepsilon<\theta \leq 1 / 2+\varepsilon . \\ \frac{2}{3-6 \varepsilon} & \text { if } \theta>1 / 2+\varepsilon\end{cases}
$$

For each level of $\varepsilon$, the distribution defines three classes of individuals with equal weight (i.e., support times density equals $1 / 3$ for each group); see Figure 5 . When $\varepsilon=1 / 6$ the distribution is uniform; when $\varepsilon$ increases, it becomes more polarized.

Observe that the distribution is symmetric so that we always have $\widehat{\theta}=$ $1 / 2$. From $(27)$, median wage and capital incomes are given by $w(\widehat{\theta})=$ $1 / 4, r(\widehat{\theta})=1 / 32$. Table 1 provides some additional characteristics of the distribution for the levels of $\varepsilon$ considered below.

\footnotetext{
${ }^{17}$ This case is not limited to having $\widehat{\theta}>\widetilde{\theta}$. Recall that this condition is sufficient but not necessary for the median individual to be decisive; see Section 4.
} 


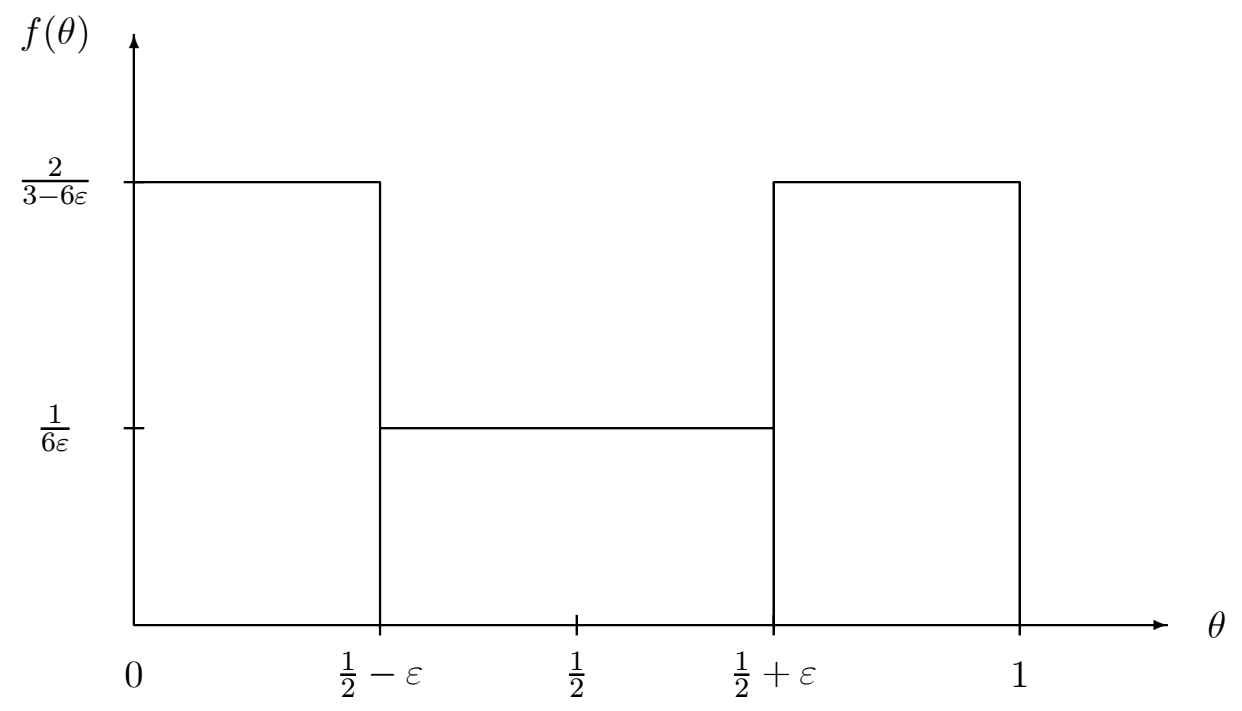

Figure 5: Distribution of types.

\begin{tabular}{|c|c|c|c|}
\hline$\varepsilon$ & $\bar{w}$ & $\bar{r}$ & $\tilde{\theta}$ \\
\hline $1 / 6$ & $1 / 3$ & $1 / 6$ & 0.790 \\
0.27 & 0.359 & 0.213 & 0.839 \\
0.275 & 0.361 & 0.215 & 0.841 \\
0.3 & 0.369 & 0.229 & 0.853 \\
\hline
\end{tabular}

Table 1: Four values of $\varepsilon$ and the corresponding values for $\bar{w}, \bar{r}$, and $\widetilde{\theta}$. 


\begin{tabular}{|l|c||c|c|}
\hline \multicolumn{1}{|c|}{$\varepsilon$} & $\begin{array}{c}q^{*}(\widehat{\theta}) \\
{\left[\alpha^{*}(\widehat{\theta})=1\right]}\end{array}$ & $\begin{array}{c}\text { Sequential vote } \\
(\alpha \text { then } q)\end{array}$ & Simultaneous vote \\
\hline $1 / 6$ & 1.838 & $39.84 \%$ & $42.2 \%$ \\
\hline 0.27 & 1.804 & $49.00 \%$ & $49.8 \%$ \\
\hline 0.275 & 1.801 & $49.70 \%$ & $50.1 \%$ \\
\hline 0.3 & 1.793 & $51.10 \%$ & $52.0 \%$ \\
\hline
\end{tabular}

Table 2: Candidate equilibrium and the maximal opposition.

In each case, we consider the most-preferred policy of the median individual $(\widehat{q}, \widehat{\alpha})$. Note that, with $\widehat{\theta}=1 / 2<\widetilde{\theta}, \widehat{\alpha}=1$ (see Proposition 2 ), and that the median individual is not necessarily decisive (see Proposition 3 ). Subsequently, we numerically determine the maximal opposition which can arise against this solution. Formally, the opposition is the proportion of individuals who support a deviation from $(\widehat{q}, \widehat{\alpha})$; the maximal opposition corresponds to the deviation which is supported by the highest number of individuals. We determine this maximum opposition both for an unrestricted simultaneous vote and for a sequential ( $\alpha$ then $q)$ vote. In either case, $(\widehat{q}, \widehat{\alpha})$ is the equilibrium if (and only if) the maximum opposition is less than $50 \%$.

The results of the simulations are summarized in Table 2. They establish the main point emphasized in the previous section; namely, that the candidate equilibrium may or may not be a Condorcet winner. Specifically, we have a candidate equilibrium in the first three cases (for $\varepsilon$ equal to $1 / 6$, 0.27 and 0.275 ) but a Condorcet winner in the first two cases. This also shows that the existence of a Condorcet winner is not just a possibility; it arises for a non-trivial set of parameter values. The last case $(\varepsilon=0.3)$ provides an illustration for Proposition 7: The median-preferred policy does not pass the candidate equilibrium's test; hence it cannot be a Condorcet winner.

To get a better understanding of these results, it is interesting to take a closer look at the determination of the degree of opposition against the 
median-preferred outcome. Consider first the case where voting takes place simultaneously over $q$ and $\alpha$. Starting with the median individual's preferred policy, we look for a direction that would be favored by a majority of voters. ${ }^{18}$ Since $(\widehat{q}, \widehat{\alpha})$ is preferred by a majority of voters to any other policy with $\alpha=1$, a deviation can be favored by a majority of voters only if it involves a decrease in $\alpha$. Furthermore, any decrease in $\alpha$ accompanied by an increase in $q$ will be rejected by all individuals with $\theta<\widehat{\theta}$, and thus can not defeat the policy $(\widehat{q}, \widehat{\alpha})$. Consequently, we have to consider deviations involving a decrease in both $\alpha$ and $q$. Such deviations could appeal both to low $\theta$ 's (because of the decrease in $q$ ) and to high $\theta$ 's (because of the decrease in $\alpha$ ). Such a direction is drawn in Figure 6.

Increasing $\varepsilon$ leads to a more polarized society, with a greater proportion of individuals favoring such deviations from the median's preferred policy. For $\varepsilon$ sufficiently low $(\varepsilon \leq 0.27$, approximately), the political support for any South West deviation is less than one half, and $(\widehat{q}, \widehat{\alpha})$ is the Condorcet winner. For $\varepsilon$ high enough $(\varepsilon \geq 0.275)$, a majority of individuals prefer the deviation and there is no Condorcet winner.

Next, consider the case where voting takes place sequentially: first over $\alpha$ and then over $q$. Observe that the set of possible deviations is smaller than in the simultaneous vote case: once $\alpha$ has been chosen, second stage voting results in $q^{E}(\alpha)$. Consequently, the maximum opposition for the sequential vote is smaller than (or equal to) that for the simultaneous vote. It is also increasing with the polarization of the distribution of voters. This is because voters opposing the median's favorite policy are to be recruited among the individuals with extreme values of $\theta$; see Figure 3a. Table 2 shows that, for $\varepsilon \leq 0.27,(\widehat{q}, \widehat{\alpha})$ is both an equilibrium in the sequential vote and a Condorcet winner. For $\varepsilon=0.275$, the median preferred point continues to

\footnotetext{
${ }^{18}$ We can come as close as we wish from a $50 \%$ support for a deviation consisting in slightly modifying $q$ while keeping $\alpha$ constant. On the other hand, such a deviation will never be preferred by a majority of voters. We thus concentrate on deviations that a majority of voters can potentially support and report the maximum opposition among such deviations in Table 2.
} 


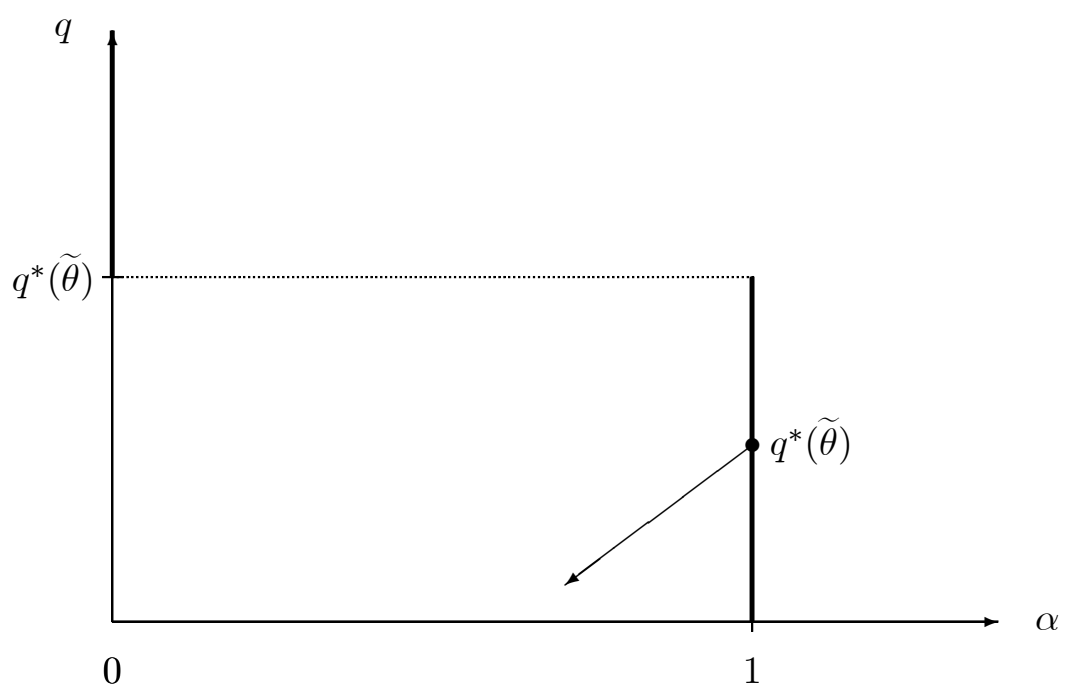

Figure 6: Candidate equilibrium and the direction of opposition.

be an equilibrium in the sequential voting procedure, even though it is not a Condorcet winner anymore. Finally, when $\varepsilon=0.3,(\widehat{q}, \widehat{\alpha})$ is no longer an equilibrium in the sequential vote.

\section{Concluding remarks}

This paper has studied how environmental policies are determined through the political process. It has considered policies that differ in two dimensions: a tax levied on polluting goods and a budgetary rule specifying the proportion of the tax proceeds that goes to wage earners and to capital owners. It has examined a number of voting procedures, sequential and simultaneous (restricted and unrestricted) for determining the values of these two parameters. Surveying the results, we conclude the paper by making two final observations about our findings.

First, the most striking feature of the results is that the best-preferred policy of the median-endowed individual constitutes a rather robust equilibrium outcome under a wide array of majority voting procedures. This fact 
notwithstanding, the paper has shown that there also exist circumstances under which an equilibrium may not exist (simultaneous voting), or if it does, it will not be the median's most-preferred policy (when voting first on the budgetary rule).

Second, our results on unrestricted simultaneous voting have an interesting implication from a methodological perspective. The existence of a Condorcet winner has been deemed as an extremely rare phenomenon in the literature on multidimensional voting. The reason for this is that the feasible voting options are invariably assumed to be an open set. When the feasible set is closed and the voters have corner preferences, the existence no longer appears that problematic. This is borne out by the widespread existence of the Condorcet winner in our setting. These two characteristics are not that rare and show up in other economic problems - at least when individuals have fixed endowments. ${ }^{19}$ To the extent that this is the case, our results point to the possibility of solving a two-dimensional voting problem for a non-trivial set of economic problems.

\footnotetext{
${ }^{19}$ This is the case, for example, when individuals choose the degree of capital and labor income taxes in a general equilibrium setting (DeDonder (2000)). It is also the case when individuals vote over non distortionary quadratic income tax functions (De Donder and Hindriks (2002)).
} 


\section{Appendix}

Proof of Lemma 1: To prove (i), differentiate (8) partially with respect to $\theta$. The result follows from the assumptions that $r^{\prime}(\theta)>0$ and $w^{\prime}(\theta)>0$. To prove (ii), differentiate (8) partially with respect to $\alpha$. This yields (10) proving the claim.

To prove (iii), rewrite (10) as

$$
\delta_{\alpha}(\theta)=\frac{r(\theta)}{\bar{w}}\left[\frac{w(\theta)}{r(\theta)}-\frac{\bar{w}}{\bar{r}}\right] .
$$

Thus $\delta_{\alpha}(\theta)$ will always have the same sign as $w(\theta) / r(\theta)-\bar{w} / \bar{r}$. From the assumption that $w(0) / r(0)>\bar{w} / \bar{r}>w(1) / r(1)$, it then follows that $\delta_{\alpha}(0)>$ 0 and $\delta_{\alpha}(1)<0$. Consequently, given the continuity of $w(\theta) / r(\theta)$, there must exist a value of $\theta$, say $\widetilde{\theta}$, at which $\delta_{\alpha}(\widetilde{\theta})=0$. Now because $w(\theta) / r(\theta)$ is decreasing in $\theta$, it also follows from (A1) that $\delta_{\alpha}(\theta)$ changes sign only once so that $\tilde{\theta}$ is unique. Additionally, this implies that $\delta_{\alpha}(\theta)>0$ as long as $\theta<\widetilde{\theta}$, and $\delta_{\alpha}(\theta)<0$ whenever $\theta>\widetilde{\theta}$.

Proof of Proposition 2 (ii): The Pigouvian tax rate $q^{F}-1$ maximizes aggregate welfare given by

$$
W^{F}=\int_{0}^{1} v(q, I(\theta), Y) f(\theta) d \theta=a(q)+\bar{m}+(q-1) y(q)-\varphi(y(q)) .
$$

Differentiating (A2) with respect to $q$ results in,

$$
\frac{\partial W^{F}}{\partial q}=[1-\delta(\theta, \alpha)] R^{\prime}(q)+\frac{\partial V}{\partial q},
$$

where we have made use of (12). Finally, evaluating (A3) at $q=q^{\dagger}(\theta ; \alpha)$ yields

$$
\frac{\partial W^{F}}{\partial q}=[1-\delta(\theta, \alpha)] R^{\prime}\left(q^{\dagger}(\theta ; \alpha)\right) .
$$

Equation (A4) tells us that whether individual $\theta$ prefers a higher or a lower tax than the Pigouvian rate depends on the proportion of taxes that he gets back in refunds. Formally, it follows from (A4) that

$$
q^{\dagger}(\theta ; \alpha) \gtreqless q^{F} \Leftrightarrow \delta(\theta, \alpha) \gtreqless 1 \text {. }
$$


In turn, evaluating (A5) at $\alpha=\alpha^{*}(\theta)$ yields

$$
q^{*}(\theta)=q^{\dagger}\left(\theta ; \alpha^{*}(\theta)\right) \gtreqless q^{F} \Leftrightarrow \delta\left(\theta, \alpha^{*}(\theta)\right) \gtreqless 1 .
$$

To establish (20), one then makes use of equations (8), (A6)-(19) and Lemma 1 (iii).

Proof of Lemma 3: Using (1), (3), (4) and the fact that $g_{w} \bar{w} / g_{r} \bar{r}=$ $\alpha /(1-\alpha)$ (obtained from (6)), the utility of individual $\theta$, as a function of $q$ and $g_{r}$, can be written as

$$
U\left(q, \alpha, g_{r}, \theta\right)=a(q)+\left(1+g_{r}\right) r(\theta)+\left(1+\frac{\alpha}{1-\alpha} g_{r} \frac{\bar{r}}{\bar{w}}\right) w(\theta)-\varphi(y(q)) .
$$

Note that (A7) does not make use of (5), i.e. of the government's budget constraint. It specifies the preferences as a function of $\alpha, q$ and $g_{r}$. It satisfies Gans and Smart's (1996) sufficient condition for the median individual to be decisive (in the vote over $q$, for any given value of $\alpha$ ). This condition relies on the Spence-Mirrlees "single-crossing" property. Applied to our setting, it requires that the marginal rates of substitution in the space $\left(q, g_{r}\right)$ be monotonic in voters' type $\theta$. Now one can easily check that the marginal rate of substitution of individual $\theta$ in the space $\left(q, g_{r}\right)$

$$
-\frac{\partial U\left(q, \alpha, g_{r}, \theta\right) / \partial g_{r}}{\partial U\left(q, \alpha, g_{r}, \theta\right) / \partial q}=\frac{r(\theta)+\frac{\alpha}{1-\alpha} \frac{\bar{r}}{\bar{w}} w(\theta)}{-a^{\prime}(q)+\varphi^{\prime}(y(q)) y^{\prime}(q)}
$$

is monotonically increasing in $\theta$ whatever the values of $\alpha, q$ and $g_{r}$. Hence, the median individual is decisive when voting on $\left(q, g_{r}\right)$ which, given the degree of freedom lost when incorporating (5), amounts to a vote on $q$.

Proof of Proposition 7: Let $\left(q^{C W}, \alpha^{C W}\right)$ denote the unrestricted Condorcet winner, i.e. the pair that is preferred by a majority of voters to any other feasible pair $(q, \alpha)$, with $q>0$ and $\alpha \in[0,1]$. We first prove that $\left(q^{C W}, \alpha^{C W}\right)$ is a Shepsle equilibrium. Fix $\alpha=\alpha^{C W}$ and let individuals vote over $q$. Because $\left(q^{C W}, \alpha^{C W}\right)$ is preferred by a majority to any pair $\left(q>0, \alpha^{C W}\right)$, the outcome of this vote is $q^{C W}$. Symmetrically, fixing 
$q=q^{C W}$ and voting over $\alpha$ gives as outcome $\left(q^{C W}, \alpha^{C W}\right)$. This pair is thus a Shepsle equilibrium.

Second, we show that $\left(q^{C W}, \alpha^{C W}\right)$ is also the outcome of a sequential voting game. Suppose that vote takes place first over $\alpha$ and then over $q$. Solving this game by backward induction, we note, by the argument explained above, that the outcome of the second step is $q^{C W}$ if the first step outcome is $\alpha^{C W}$. Going back to the first step, individuals then realize that a vote for $\alpha^{C W}$ is indeed a vote for $\left(q^{C W}, \alpha^{C W}\right)$. Since this pair is preferred by a majority to any other pair, $\alpha^{C W}$ is the majority voting equilibrium in the first stage, and $q^{C W}$ in the second stage. Note also that this line of reasoning applies mutatis mutandis to the other sequential vote (first $q$ then $\alpha)$. 


\section{References}

Besley, B., Coate, S., 1997, An economic model of representative democracy. Quarterly Journal of Economics 112, 85-114.

Cremer, H., P. De Donder and F. Gahvari, 2001, Political sustainability and the design of environmental taxes, mimeo.

Davis, O., DeGroot, M., Morris, M., 1972, Social preference orderings and majority rule, Econometrica 40, 147-157.

De Donder P, 2000, Voting on Taxation in a Simple General equilibrium Model, mimeo University of Toulouse.

De Donder, P and J. Hindriks, 2002, Majority Support for Progressive Income Taxation: The Importance of Corner Preferences, mimeo University of Toulouse.

Gans, J.S. and M. Smart, 1996, Majority voting with single crossing preferences. Journal of Public Economics 59, 219237.

Guesnerie, R. and M. Jerison, 1991, Taxation as a Social Choice Problem. The Scope of the Laffer Argument, Journal of Public Economics, 44, 37-64.

Osborne, M.J., A. Slivinski, 1996, A Model of Political Competition with Citizen candidates, Quarterly Journal of Economics, 111, 65-96.

Plott, C., 1967, A notion of equilibrium and its possibility under majority rule, American Economic Review 57, 787-806.

Shepsle, K., 1979, Institutional arrangements and equilibrium in multidimensional voting models. American Journal of Political Science 23, 27-59.

Schoffield, N., Tovey, C., 1992, Probability and convergence for 
supramajority rule with Euclidean preferences, Mathematical and Computer Modeling 16, 41-58.

Tovey, C., 1992, The probability of an undominated central voter in two-dimensional majority voting, Social Choice and Welfare $9,43-48$. 